\title{
Lunasin Inhibits Cell Proliferation via Apoptosis and Reduces the Production of Proinflammatory Cytokines in Cultured Rheumatoid Arthritis Synovial Fibroblasts
}

\author{
Shaohui Jia, ${ }^{1}$ Shufang Zhang, ${ }^{2,3}$ Hong Yuan, ${ }^{2}$ and Ning Chen ${ }^{1}$ \\ ${ }^{1}$ College of Health Science, Hubei Provincial Collaborative Innovation Center for Exercise and Health Promotion, \\ Wuhan Sports University, Wuhan 430079, China \\ ${ }^{2}$ Graduate School, Wuhan Sports University, Wuhan 430079, China \\ ${ }^{3}$ College of Sports Science and Technology, Wuhan Sports University, Wuhan 430205, China
}

Correspondence should be addressed to Ning Chen; nchen510@gmail.com

Received 1 September 2014; Revised 6 January 2015; Accepted 6 January 2015

Academic Editor: Shigeru Kotake

Copyright ( 2015 Shaohui Jia et al. This is an open access article distributed under the Creative Commons Attribution License, which permits unrestricted use, distribution, and reproduction in any medium, provided the original work is properly cited.

\begin{abstract}
Lunasin, a peptide with 43 amino acid residues and initially isolated and identified in soybean cotyledon, has gained extensive attention due to its anti-inflammatory and anticancer properties. However, its treatment efficacy on rheumatoid arthritis (RA) and corresponding mechanisms have not been reported. Herein, the synovial fibroblasts harvested and isolated from patients with RA were treated with lunasin at various concentrations to examine the proliferation, apoptosis status, and corresponding cell cycle of cultured RA synovial fibroblasts. Meanwhile, the underlying mechanisms of lunasin for RA treatment are explored through Western blot, real-time PCR, ELISA, and luciferase reporter assays. Lunasin significantly inhibited the proliferation and induced the apoptosis of cultured RA synovial fibroblasts. In addition, lunasin reduced the production of interleukin-6 (IL-6), IL-8, and matrix metalloproteinase-3 (MMP-3) and suppressed the activation of NF- $\kappa$ B in cultured RA synovial fibroblasts but did not reveal obvious modulation on the secretion and gene expression of MMP-1. Therefore, lunasin will have promising potential as a novel nutritional supplement or drug candidate for RA due to its potency of suppressing synovial cell proliferation and decreasing the production of proinflammatory cytokines and MMPs in synovial cells.
\end{abstract}

\section{Introduction}

Rheumatoid arthritis (RA) is a systematic inflammatory disease and afflicts $0.5-1.0 \%$ of population all over the world $[1,2]$. RA is characterized by inflammatory changes of synovial tissues and the formation of rheumatoid pannus that results in the erosion of adjacent cartilage and subchondral bone, thus subsequently causing irreparable joint destruction. Pathological studies show that synovial fibroblasts play a critical role in the pathogenesis of RA [3]. The markedly increases of synovial fibroblasts in RA have been reported in previous studies [4] and the proliferation of synovial fibroblasts has been thought to contribute to the formation of rheumatoid pannus [5]. Moreover, RA synovial fibroblasts can spontaneously secrete numerous proinflammatory cytokines such as interleukin-6 (IL-6) and IL-8 and matrix metalloproteinases (MMPs) including MMP-1 and MMP-3, which plays an important role in progressive destruction of articular cartilage and bone [6-9]. Currently, many natural compounds such as celastrol [10], sinomenine [11], and curcumin [12] derived from plants or plant extracts such as Periploca sepium extract [13] and Tripterygium wilfordii Hook. F. extract [14] have reported to treatment RA through suppressing the expression of proinflammatory cytokines or mediators, adhesion molecules and MMPs in synovial fibroblasts, the peptide-based agents from natural plants for inhibiting these cell signaling molecules or cell signaling pathways in preclinical or clinical investigations are less reported.

Lunasin is a 43-amino acid peptide isolated and identified from soybean and other plant sources [15]. Lunasin contains nine aspartic acid residues on its carboxyl terminal that 
have been found to be responsible for its antimitotic effect [16]. It also contains a cell adhesion motif composed of arginine-glycine-aspartic acid (RGD) residues to allow the attachment to the extracellular matrix and a predicated helix with structural homology to a conserved region of chromatin-binding proteins [17]. Earlier in vitro studies have shown that lunasin can exert chemopreventive properties in mammalian cells elicited by chemical carcinogens and viral oncogenes $[18,19]$. Moreover, lunasin has been reported to reduce the tumor incidence of skin cancer and breast cancer in mouse models $[20,21]$. Recent studies have begun to unmask the antioxidant and anti-inflammatory potentials of lunasin through inhibiting different inflammatory mediators in macrophage cell line RAW 264.7 [22, 23]. The pathogenesis of RA is mostly similar as tumors and mainly characterized by inflammatory change and the proliferation of synovial fibroblasts. Therefore, we speculate that lunasin may be benefit for RA because of its anti-inflammatory potency. In order to explore the treatment efficacy of lunasin for RA and underlying mechanisms, we investigated the effect of lunasin on the progression of RA using human synovial fibroblasts from knee joint of patients with RA as an in vitro model, which will provide the promising potential or treatment strategy of lunasin for RA as a novel supplement and drug candidate.

\section{Materials and Methods}

2.1. Polypeptide and Reagents. The polypeptide lunasin was synthesized by Senggong Company (Shanghai, China). 3-(4, 5-Dimethylthiazol-2-yl)-2,5-diphenyltetrazolium bromide (MTT), propidium iodide (PI), and Triton X-100 were purchased from Sigma Chemical Company (St. Louis, MO, USA). The Annexin V-fluorescein isothiocyanate (FITC)/PI apoptosis detection kit was obtained from Beyotime Institute of Biotechnology (Shanghai, China). All primary antibodies were purchased from Santa Cruz Biotechnology (Santa Cruz, CA, USA).

2.2. Isolation and Culture of Synovial Cells. Synovial tissues were harvested from six patients undergoing knee replacement surgeries owing to their RA in Tongji Hospital (Wuhan, China). The patients were provided with the informed consent for research and the experimental protocols were reviewed and proved by Institutional Review Board at Tongji Hospital, Huazhong University of Science and Technology. The scores of all patients are higher than 7.

Synovial fibroblasts were isolated by sequential digestion of the dissected synovial tissues with type I collagenase and cultured in Dulbecco Modified Eagle Medium (DMEM) (Gibco, Grand Island, NY, USA) at $37^{\circ} \mathrm{C}$ in a humidified atmosphere with $5 \% \mathrm{CO}_{2}$, supplemented with $10 \%(\mathrm{v} / \mathrm{v})$ fetal bovine serum (FBS), $100 \mathrm{U} / \mathrm{mL}$ penicillin, and $100 \mathrm{mg} / \mathrm{L}$ streptomycin.

2.3. Cytokine Assay. Cells $\left(1 \times 10^{6}\right.$ cells/well $)$ were plated in 6-well cell culture plates overnight and then incubated with lunasin at designed concentrations $(0,10,50,100$, and
$200 \mu \mathrm{M})$ for $72 \mathrm{~h}$. After treatment, cell supernatants were collected to analyze the secretion of cytokines (IL-6, IL-8, MMP-1, and MMP-3) using enzyme linked immunosorbent assay (ELISA) kit (R\&D Systems Inc., Minneapolis, MN, USA).

2.4. Cell Proliferation Assay. For cell proliferation assay, synovial fibroblasts were plated in 96-well plates at a density of $2.5 \times 10^{3}$ cells per well and allowed to synchronize by incubation in serum-free DMEM medium for $48 \mathrm{~h}$. Then, the cell culture medium was replaced by normal DMEM medium containing lunasin at varying concentrations $(0,10$, $50,100$, and $200 \mu \mathrm{M})$. Cells were counted at $0,24,48$, and $72 \mathrm{~h}$, and cell number was evaluated by crystal violet staining as described as follows: cells were fixed with $1 \%$ glutaraldehyde and stained with $0.1 \%$ crystal violet. The unbound dye was dissolved with $0.2 \%$ Triton X-100. Light enhancement linearly correlated with the cell number was analyzed at $570 \mathrm{~nm}$ using a fluorescence microplate reader (Tecan Sunrise, Salzburg, Austria).

2.5. Cell Cycle Analysis. The distribution of cell cycle was detected by flow cytometry according to the manufacturer's protocol. Briefly, synovial fibroblasts were seeded in 6-well plates at a density of $1 \times 10^{6}$ cells/well. After adhesion, cells were starved for $48 \mathrm{~h}$ in serum-free DMEM medium. Then, the medium was replaced by fresh normal DMEM medium in the presence or absence of lunasin. After incubation for another $48 \mathrm{~h}$, the cells were collected and fixed in $70 \%$ icecold ethanol. The cell samples were resuspended in $200 \mu \mathrm{L}$ of solution containing $50 \mu \mathrm{g} / \mathrm{mL}$ PI, $0.1 \mathrm{mg} / \mathrm{mL}$ RNase A, and $0.1 \%$ Triton X-100 and then incubated at $37^{\circ} \mathrm{C}$ for $30 \mathrm{~min}$. Finally, the cell cycle analysis was performed on a flow cytometer (Beckman Coulter, Brea, CA, USA).

2.6. Apoptosis Analysis. Synovial fibroblasts in 6-well plates $\left(1 \times 10^{6}\right.$ cells/well $)$ were treated with lunasin at various concentrations $(0,10,50,100$, and $200 \mu \mathrm{M})$ for $48 \mathrm{~h}$, and $60 \mu \mathrm{M}$ $\mathrm{H}_{2} \mathrm{O}_{2}$ was used to induce apoptosis as the positive control. At the end of treatment, the cells were collected and washed with PBS for three times. Then, the cells were resuspended with $195 \mu \mathrm{L}$ of FITC-binding buffer and incubated with $5 \mu \mathrm{L}$ of Annexin V-FITC for $15 \mathrm{~min}$ at room temperature in a dark environment. After washed with PBS, the cells costained with PI solution were subjected to flow cytometric analysis.

2.7. RT-PCR Analysis. Total RNA was isolated using TRIzol reagent (Invitrogen, Carlsbad, CA, USA) and reversely transcribed to cDNA using a RevertAid cDNA Synthesis Kit (Fermentas International Inc., Vilnius, Lithuania) according to the manufacturer's instructions. The primer sequences and reaction conditions of IL-6, IL-8, MMP-1, MMP-3, and GAPDH were shown in Table 1. The PCR products were evaluated by $2 \%$ agarose gel electrophoresis.

2.8. Western Blot Analysis. After treatment, cell samples were lysed in $20 \mu \mathrm{L}$ of cell lysis buffer containing $1 \mathrm{~mm}$ phenylmethanesulfonyl fluoride (PMSF). The extracted proteins 
TABLE 1: Primer sequences and reaction conditions of RT-PCR.

\begin{tabular}{|c|c|c|c|c|}
\hline Genes & Primers & Sequences $\left(5^{\prime}-3^{\prime}\right)$ & Annealing temperature $\left({ }^{\circ} \mathrm{C}\right)$ & Cycle number \\
\hline \multirow{2}{*}{ IL-6 } & Forward & GGCTGCTTCTGGTGATGG & \multirow{2}{*}{55} & \multirow{2}{*}{30} \\
\hline & Reverse & AGAGATTTTGCCGAGGATGTA & & \\
\hline \multirow{2}{*}{ IL-8 } & Forward & GCCAAGGAGTGCTAAAGAACTTAGA & \multirow{2}{*}{58} & \multirow{2}{*}{30} \\
\hline & Reverse & ATTTCTGTGTTGGCGCAGTGT & & \\
\hline \multirow{2}{*}{ MMP-1 } & Forward & AGGGTCAAGCAGACATCA & \multirow{2}{*}{56} & \multirow{2}{*}{30} \\
\hline & Reverse & CAGAAGGGCAAGCATTAG & & \\
\hline \multirow{2}{*}{ MMP-3 } & Forward & CCTGCTTTGTCCTTTGATGC & \multirow{2}{*}{55} & \multirow{2}{*}{30} \\
\hline & Reverse & TGAGTCAATCCCTGGAAAGTC & & \\
\hline \multirow{2}{*}{ GAPDH } & Forward & CAAGGTCATCCATGACAACTTTG & \multirow{2}{*}{56} & \multirow{2}{*}{25} \\
\hline & Reverse & GTCCACCACCCTG TTGCTGTAG & & \\
\hline
\end{tabular}

were separated by $17 \%$ SDS-PAGE and transferred to PVDF membranes by $2 \mathrm{~h}$ electroblotting. Blots were blocked in $5 \%$ nonfat dry milk for $1 \mathrm{~h}$ at room temperature and then incubated at $4^{\circ} \mathrm{C}$ overnight with primary antibodies. Membranes were washed with TBS containing $0.05 \%$ Tween-20 (TBS$\mathrm{T}$ buffer) for 3 times and then incubated with horseradish peroxidase-conjugated secondary antibodies at $37^{\circ} \mathrm{C}$ for $1 \mathrm{~h}$. Finally, the blots were developed with the enhanced chemiluminescence (ECL) kit (Pierce Biotechnology, Rock-ford, IL, USA).

2.9. Luciferase Reporter Assay. The activity of nuclear factor kappa-light-chain-enhancer of activated B cells (NF- $\kappa \mathrm{B})$ was tested as previous description [24]. Briefly, the vector of pNF- $\kappa$ B-Luc (Stratagene Inc., La Jolla, CA, USA) containing Photinus pyralis (firefly) luciferase reporter gene and the vector of phRL-TK vector (Promega, Madison, WI, USA) containing Renilla luciferase reporter gene were cotransfected into synovial fibroblasts using lipofectamine 2000 reagent. The transfected cells were simultaneously challenged with IL- $1 \beta$ and lunasin for $24 \mathrm{~h}$. After treatment, the cell samples were collected and the activity of NF- $\kappa \mathrm{B}$ in cell samples was analyzed by TD-20/20 luminometer (Turner BioSystems, Sunnyvale, CA, USA) with a dual-luciferase reporter assay system (Promega, Madison, WI, USA).

2.10. Statistical Analysis. All experiments were repeated at least 3 times. Data were expressed as mean \pm SD and analyzed by Student's $t$-test through GraphPad Prism Version 5.0 software (GraphPad Software Inc., San Diego, CA, USA). The statistically significant difference was considered at $P<0.05$.

\section{Results}

3.1. Lunasin Inhibits the Proliferation of Synovial Fibroblasts. The effect of lunasin on the proliferation of synovial fibroblasts was evaluated by crystal violet staining. As shown in Figure 1, lunasin significantly repressed the proliferation of synovial fibroblasts in a dose- and time-dependent manner. After $72 \mathrm{~h}$ incubation with lunasin at the concentrations of $100 \mu \mathrm{M}$ and $200 \mu \mathrm{M}$, the cell number revealed a decrease by $44.7 \pm 3.2 \%$ and $41.4 \pm 2.0 \%$, respectively, when compared with the control. In addition, lunasin treatment at a lower

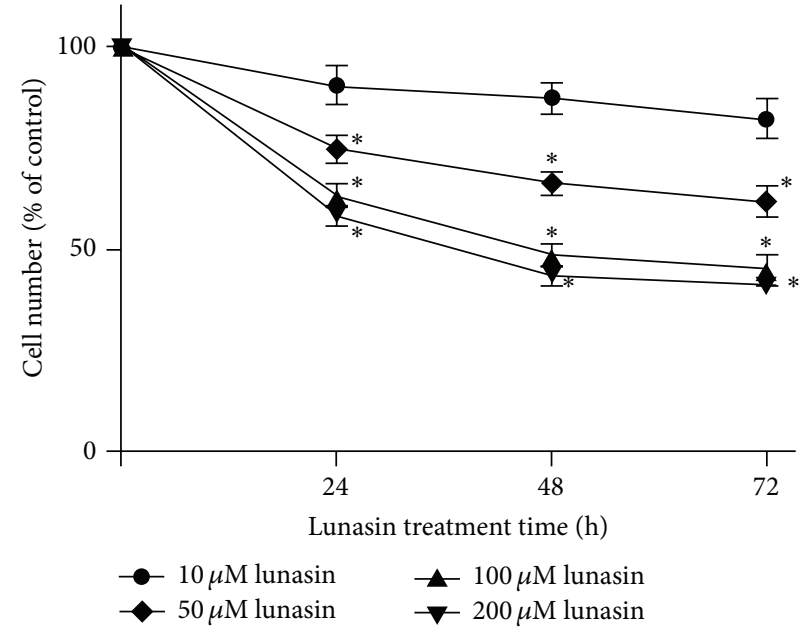

FIGURE 1: Lunasin inhibits the proliferation of RA synovial fibroblasts. Cells were synchronized and incubated with lunasin at various concentrations for 24,48 , and $72 \mathrm{~h}$, respectively. Then, the cell samples were harvested and subjected to count the number by crystal violet staining. The $\mathrm{IC}_{50}$ was $153.3 \pm 3.2 \mu \mathrm{M}$ after $48 \mathrm{~h}$ incubation. Data were expressed as the percentage relative to the untreated control samples, and each value was expressed as mean \pm SD for three independent experiments by GraphPad Prism Software Version 5.0. ${ }^{*} P<0.05$ was considered a significant difference when compared with the control.

dose $(50 \mu \mathrm{M})$ for $24 \mathrm{~h}$ also could significantly inhibit the proliferation of synovial fibroblasts, whereas no obvious inhibition on cell proliferation was observed when synovial fibroblasts were treated with lunasin at the concentration of $10 \mu \mathrm{M}$ even for 3 days. The $\mathrm{IC}_{50}$ value of lunasin after $48 \mathrm{~h}$ treatment was $153.3 \pm 3.2 \mu \mathrm{M}$.

3.2. Lunasin Induces G0/G1 Phase Arrest of Synovial Fibroblasts. In order to explore whether the induction of cell cycle arrest contributes to the antiproliferative potency of lunasin in synovial fibroblasts, flow cytometric analysis was carried out to evaluate the cell cycle progression. As shown in Figures 2(a) and 2(b), the treatment of synovial fibroblasts with lunasin at gradually increasing concentrations $(0,10,50,100$, 

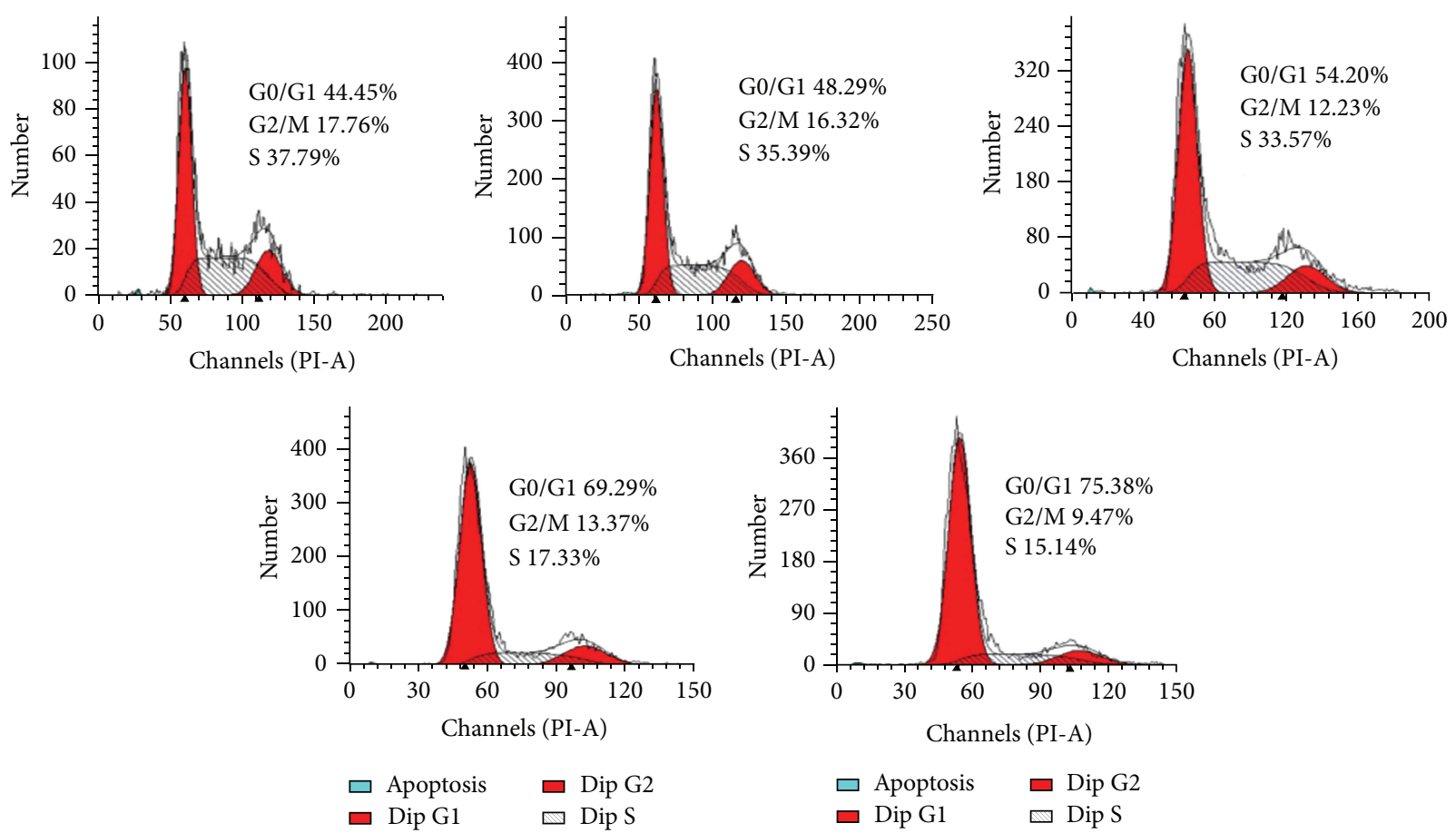

$$
\square \text { Apoptosis } \square \text { Dip G2 }
$$

(a)
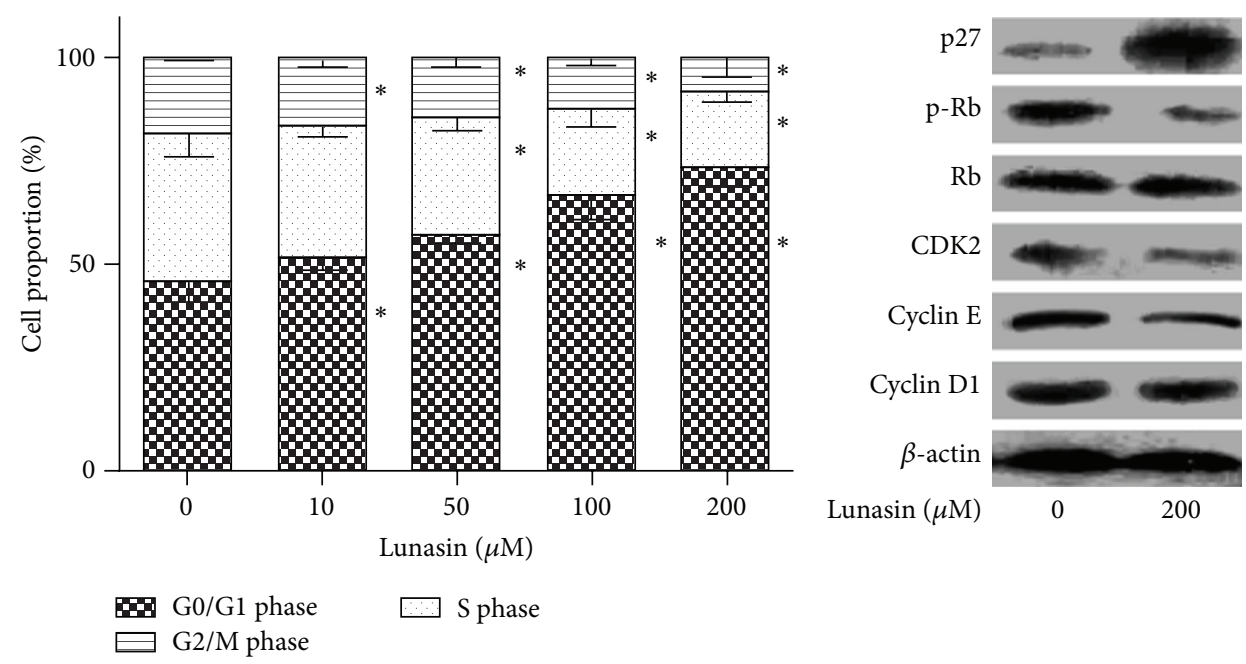

(b)

(c)

FIGURE 2: Effect of lunasin on the cell cycle of synovial fibroblasts and the expression of the proteins related to G0/G1 cell cycle arrest. (a) Cell cycle progression of synovial fibroblasts was examined by flow cytometry. Synovial fibroblasts were challenged with lunasin at concentrations of $0,10,50,100$, and $200 \mu \mathrm{M}$ for $48 \mathrm{~h}$, respectively. After treatments, the cells were collected and subjected to the analysis of cell cycle distribution by flow cytometry. (b) Quantification of the cell cycle data. Data were expressed as mean \pm SD for three independent experiments by GraphPad Prism Software Version 5.0. ${ }^{*} \mathrm{P}<0.05$ was considered a significant difference when compared with the control. (c) Synovial fibroblasts were treated with lunasin at the concentrations of 0 and $200 \mu \mathrm{M}$ for $48 \mathrm{~h}$. The cell samples were harvested and the expression of cell cycle-relevant proteins was evaluated.

and $200 \mu \mathrm{M}$ ) for $48 \mathrm{~h}$ elevated the proportion of the cells in G0/G1 phase from $45.4 \pm 2.5 \%$ up to $51.7 \pm 2.0 \%, 59.2 \pm 1.5 \%$, $66.7 \pm 3.2 \%$, and $73.3 \pm 2.4 \%$, respectively, and on the contrary reduced the proportion of the cells in S phase from $35.8 \pm$ $2.9 \%$ to $31.6 \pm 1.6 \%, 29.1 \pm 2.6 \%, 20.82 \pm 2.2 \%$, and $18.1 \pm$ $1.5 \%$, as well as decreasing the proportion of the cells in G2/M phase from $18.8 \pm 0.6 \%$ to $16.7 \pm 1.0 \%, 14.7 \pm 1.3 \%, 12.6 \pm$
$1.0 \%$, and $8.9 \pm 2.7 \%$, respectively. These results indicated that lunasin arrested synovial fibroblasts in G0/G1 phase in a dosedependent manner.

In order to further uncover the underlying mechanisms of lunasin on the inhibition of synovial fibroblast proliferation, we then examined the expressions of cell cycle-related proteins using Western blotting. Compared with the control, the 


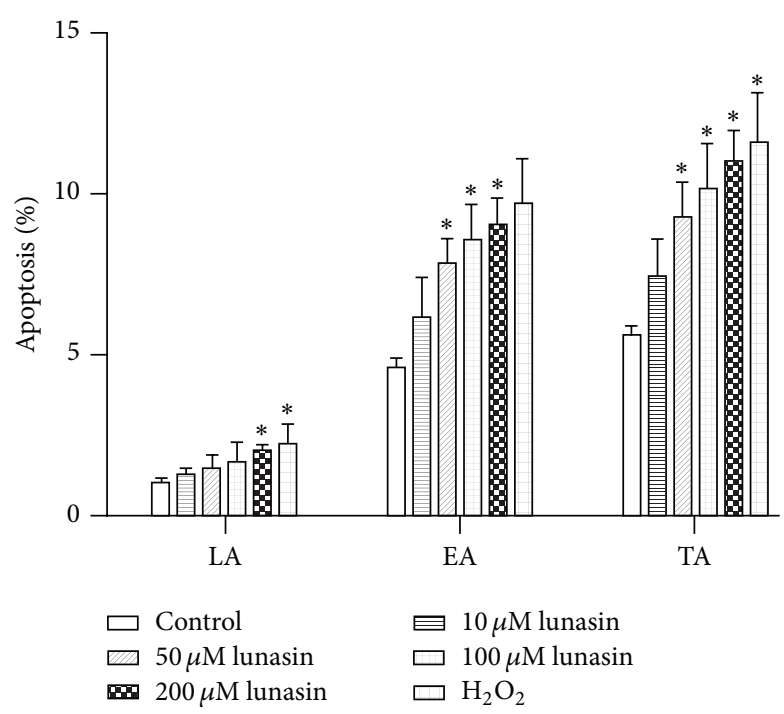

(a)

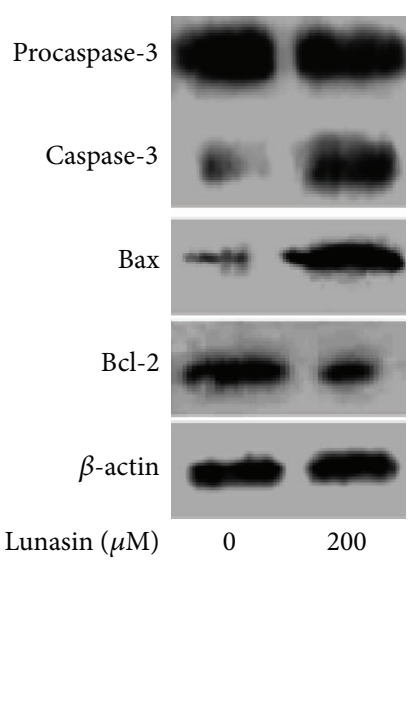

(b)

FIGURE 3: Effect of lunasin on the apoptosis of synovial fibroblasts and the expression of apoptosis-relevant proteins. (a) Synovial fibroblasts were incubated with lunasin at various concentrations $(0,10,50,100$, and $200 \mu \mathrm{M})$ for $48 \mathrm{~h}$, and then the cells were harvested and the apoptosis proportion of the cells was examined by flow cytometry using V-FITC/PI double staining. The proportion of cells in early apoptosis (positive for FITC and negative for PI, FITC ${ }^{+} / \mathrm{PI}^{-}$) and late apoptosis (positive for both FITC and PI, FITC ${ }^{+} / \mathrm{PI}^{+}$) were analyzed by flow cytometry. Data were expressed as mean $\pm \mathrm{SD}$ for three independent experiments. ${ }^{*} \mathrm{P}<0.05$ was considered a significant difference when compared with the control. (b) Synovial fibroblasts were treated with lunasin at the concentrations of 0 and $200 \mu \mathrm{M}$ for $48 \mathrm{~h}$. The cell samples were then harvested and the expression of apoptosis-relevant proteins was evaluated. LA: late apoptosis; EA: early apoptosis; TA: total apoptosis.

treatment of synovial fibroblasts with $200 \mu \mathrm{M}$ lunasin for $48 \mathrm{~h}$ significantly decreased the expression of Cyclin-dependent kinase 2 (CDK2) and Cyclin E and conspicuously reduced the phosphorylation of $\mathrm{Rb}$; in contrast, lunasin strongly elevated the level of p27 but failed to modify the production of Cyclin D1 (Figure 2(c)).

\subsection{Lunasin Promotes the Apoptosis of Synovial Fibroblasts.} In order to corroborate the possible suppression of cell proliferation by lunasin, synovial fibroblasts were doublestained with FITC and PI to examine the apoptosis by flow cytometry. Figure 3(a) shows the quantitative data for the change in proportion of apoptotic cells. The cells exposed to lunasin revealed a clear apoptosis induction in a dosedependent manner when compared with the control. After the cells incubated with lunasin at the concentrations of 0,10 , 50,100 , and $200 \mu \mathrm{M}$ for $48 \mathrm{~h}$, the proportion of early apoptosis was enhanced from $4.6 \pm 0.2 \%$ to $6.2 \pm 0.7 \%, 7.8 \pm 0.5 \%, 8.6$ $\pm 0.6 \%$, and $9.0 \pm 0.5 \%$, respectively, and the percentage of total apoptosis was elevated from $5.6 \pm 0.2 \%$ to $7.4 \pm 0.7 \%$, $9.3 \pm 0.6 \%, 10.17 \pm 0.8 \%$, and $11.0 \pm 0.6 \%$, respectively. These results suggest that lunasin is an effective inducer of apoptosis in synovial fibroblasts, especially for early apoptosis.

In order to elucidate the mechanisms of lunasin on the apoptosis of synovial fibroblasts, Western blotting was conducted to evaluate the change in the expression of apoptosisrelevant proteins. After synovial fibroblasts incubated with $200 \mu \mathrm{M}$ lunasin for $48 \mathrm{~h}$, lunasin obviously induced the expression of activated caspase- 3 and Bax; and suppressed the expression of antiapoptotic protein Bcl-2 (Figure 3(b)).
3.4. Lunasin Decreases the Production of IL-6, IL-8, and MMP3 in RA Synovial Fibroblasts. The secretion of proinflammatory cytokines (IL-6, IL-8) and MMPs play an important role in RA progression. Therefore, we investigated the effect of lunasin on the secretion of IL-6, IL-8, MMP-1, and MMP3 . In the presence of IL- $1 \beta$ induction, the secretion of IL6, IL-8, MMP-1, and MMP-3 revealed an obvious increase in the RA synovial fibroblasts. In contrast, the application of lunasin resulted in a dose-dependent reduction on the secretion of IL-6, IL-8, and MMP-3 in synovial fibroblasts induced by IL-1 $\beta$ (Figures $4(\mathrm{a})-4(\mathrm{c})$ ); however, no obvious change in the secretion of MMP-1 was observed during the application of lunasin, even at the high dose $(200 \mu \mathrm{M})$ (Figure 4(d)).

To further confirm the effect of lunasin on the production of proinflammatory factors and MMPs in synovial fibroblasts, the gene expression of IL-6, IL-8, MMP-1, and MMP-3 was investigated by RT-PCR. In agreement with the observation of ELISA assay, IL-1 $\beta$ caused a markedly upregulated gene expression of IL-6, IL-8, MMP-1, and MMP-3; however, after the exposure to lunasin, synovial fibroblasts showed a significant decrease in the gene expression of IL-6, IL-8, and MMP-3 stimulated by IL-1 $\beta$.

3.5. Lunasin Reverses IL-1 $\beta$-Induced NF- $\kappa B$ Activation in RA Synovial Fibroblasts. The activation of NF- $\kappa \mathrm{B}$ was observed in RA synovial fibroblasts and thought to be responsible for increasing the production of IL-6, IL-8, MMP-1, and MMP3 [6-9]. Therefore, we investigated whether lunasin could affect the activation of NF- $\kappa$ B. As shown in Figure $5,10 \mathrm{ng} / \mathrm{mL}$ 


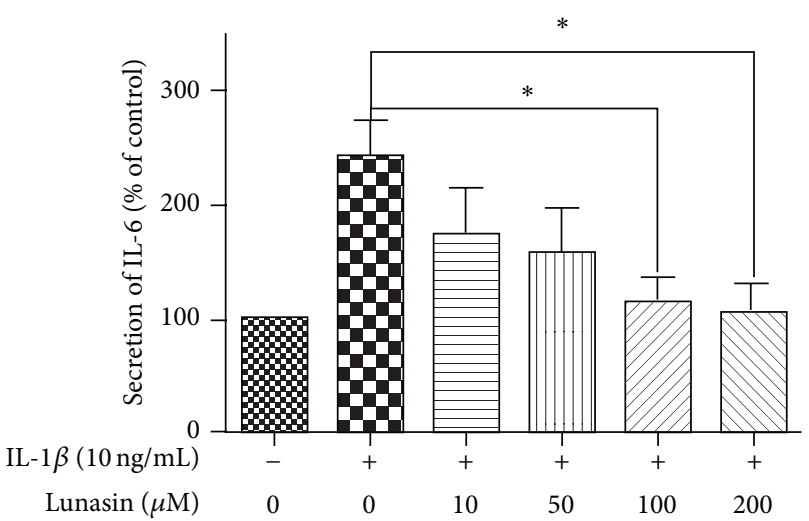

(a)

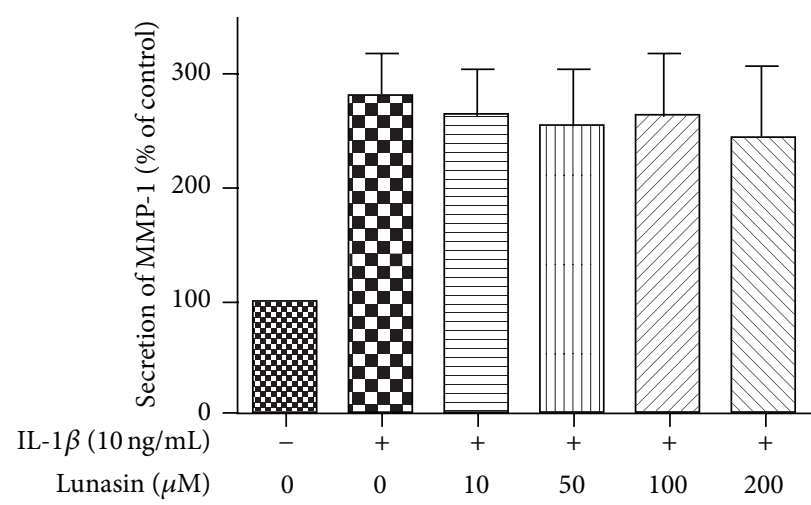

(c)

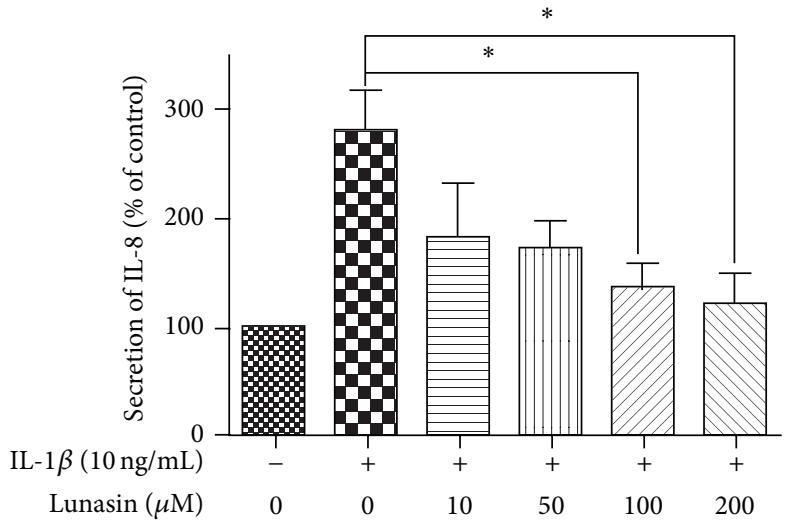

(b)

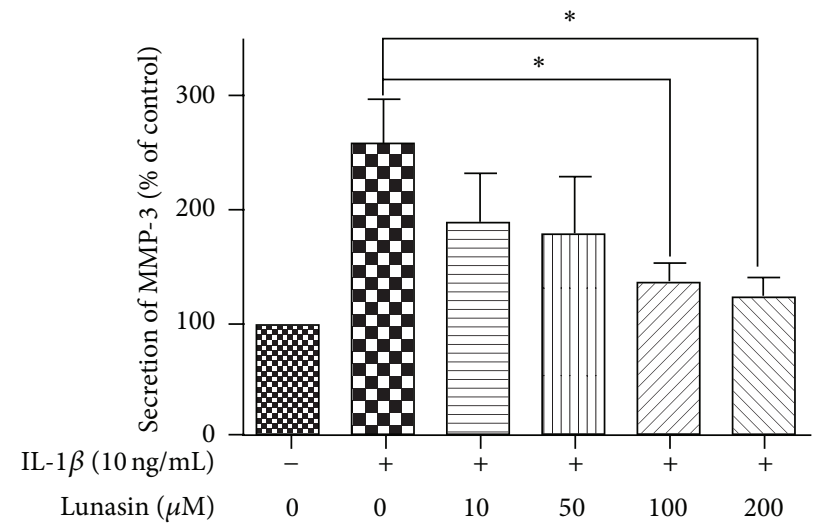

(d)

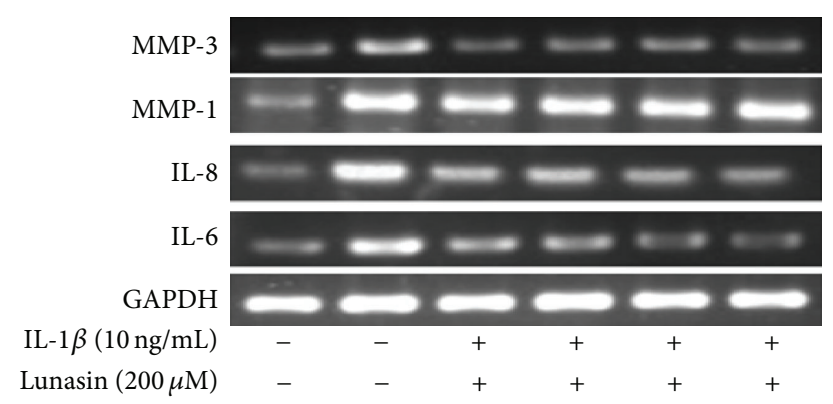

(e)

FIGURE 4: Effect of lunasin on the production of proinflammatory cytokines and MMPs. Synovial fibroblasts in the presence of IL- $1 \beta$ induction were treated with lunasin at various concentrations $(0,10,50,100$, and $200 \mu \mathrm{M})$ for $72 \mathrm{~h}$ in serum-free DMEM medium. After treatments, the cell-free medium and RA synovial fibroblasts were collected to evaluate the secretion of IL-6 (a), IL-8 (b), MMP-1 (c), and MMP-3 (d) by their corresponding ELISA kits, respectively. (e) The gene expression of IL-6, IL-8, MMP-1, and MMP-3 was examined by RT-PCR. Data were expressed as mean \pm SD for three independent experiments by GraphPad Prism Software Version 5.0. ${ }^{*} P<0.05$ was considered a significant difference when compared with the control.

IL-1 $\beta$ caused a robust increase ( 2.73 fold) in NF- $\kappa$ B activity, while lunasin revealed an inhibitory effect on the activation of NF- $\kappa \mathrm{B}$ in RA synovial fibroblasts in the presence of IL-1 $\beta$ induction.

\section{Discussion}

The hyperplasia of synovial tissue is ascribed to both an increased proliferation [25] and an impaired apoptosis of synovial fibroblasts [26]. The inhibition of synovial fibroblast proliferation is considered a potentially therapeutic approach for RA [27]. Traditional natural compounds or extracts from plants, for example, celastrol [10] or Tripterygium wilfordii Hook. F. extract [14], reveal their potential on the treatment of RA by inhibiting the proliferation RA synovial fibroblasts due to the induction of DNA damage, cell cycle arrest, and apoptosis; however, the high toxicity or unclear bioactive components in extracts largely limit their application. In 


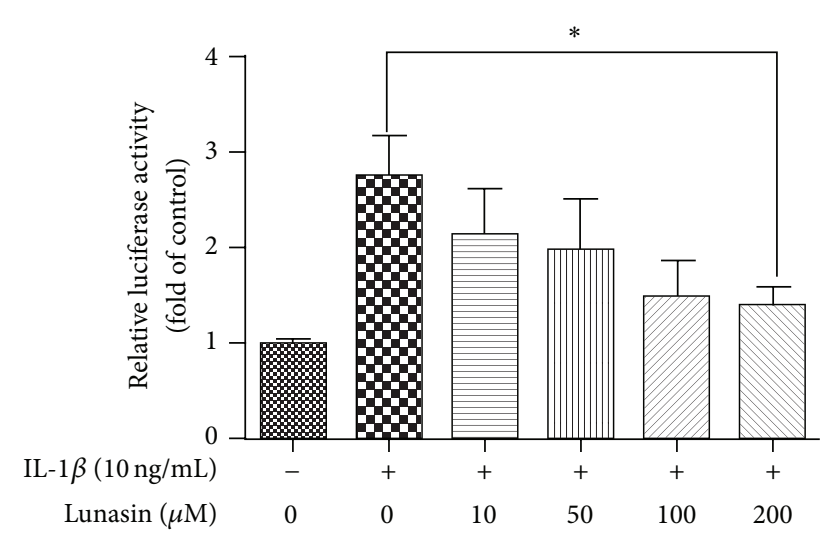

FIGURE 5: Lunasin inhibits the activation of NF- $\kappa$ B in RA synovial fibroblasts in the presence of IL- $1 \beta$ induction. Transfected synovial fibroblasts were coincubated with IL- $1 \beta$ at the dose of $10 \mathrm{ng} / \mathrm{mL}$ and lunasin at the designed concentrations for $24 \mathrm{~h}$. Then, the cell samples were collected and the activity of NF- $\kappa$ B was analyzed. Data were mean \pm SD for three independent experiments by GraphPad Prism Software Version 5.0. ${ }^{*} P<0.05$ was considered a significant difference when compared with the control.

the present study, we have found that lunasin from soybean without toxicity could significantly inhibit the proliferation of synovial fibroblasts through inducing G0/G1 phase arrests. We have also demonstrated that lunasin could promote the apoptosis of synovial fibroblasts in a dose-dependent manner. Lunasin contains a specific RGD sequence as a cell adhesion motif responsible for the attachment of lunasin to extracellular matrix, which has demonstrated the role of RGD peptide in inducing apoptosis in different cell lines via a caspase-dependent mechanism [28, 29]. Additionally, nine poly-D residues on the carboxyl termination of lunasin can specifically bind to histones, thereby affecting proper complex formation, leading to mitotic termination and eventually resulting in cell death [16], but these natural compounds including celastrol [10], sinomenine [11], and curcumin [12] extracted from plants or plant extracts containing similar small molecules such as Tripterygium wilfordii Hook. F. extract [14] can not accomplish the specific targeting function during the treatment process of RA. Previous investigations have reported that lunasin could induce the apoptosis of human colon cancer cells through the activation of mitochondrial pathway by upregulating Bax expression, decreasing $\mathrm{Bcl}-2$ expression and promoting the activation of caspase3 [30]. Since lunasin-induced apoptosis and G0/G1 cell cycle arrests in synovial fibroblasts are observed, we further investigated the effect of lunasin on the expression of proteins associated with G0/G1 cell cycle regulation and apoptosis modulation. In our study, Western blotting showed that lunasin significantly downregulated the expression of Cyclin $\mathrm{E}, \mathrm{CDK} 2$ and suppressed the phosphorylation of $\mathrm{Rb}$, as well as inhibited the expression of antiapoptotic protein Bcl-2 but elevated the production of p27, Bax, caspase-3, caspase-8, and caspase-9. Cyclin $\mathrm{E}$ is an important regulator of late $\mathrm{G1}$ phase and its activity is positively correlated with its expression [31]. Rb becomes hyperphosphorylated after the activation of
Cyclin E/CDK2; then, the phosphorylated Rb liberates E2F to generate the transcription of genes for the requirement of cell cycle progression and drives the cell into S phase [32]. P27 is a negative regulator of cell cycle and plays a crucial role in the inhibition of Cyclin/CDK complexes [33]. The data from Western blotting further confirm the inhibitory potency of lunasin on the proliferation of synovial fibroblasts.

Inflammatory changes of synovial fibroblasts also play a vital role in the progression of RA. It has been reported that IL-6 and IL-8 overexpressed in infected synovial fibroblasts. One data indicate that lunasin could inhibit the inflammation by inactivating NF- $\kappa \mathrm{B}$ pathway [34]. Moreover, the RGD peptide of lunasin has been considered to play an important role in suppressing inflammation [35]. In our study, we have also found that lunasin could significantly decrease the production of IL- 6 and IL- 8 in cultured RA synovial fibroblasts induced by IL- $1 \beta$ in a dose-dependent manner. IL6 has been reported to inhibit the proliferation of synovial fibroblasts in the presence of soluble IL-6 receptor [36]. Contrarily, another observation reveals that IL- 6 can induce the growth of synovial fibroblasts [37]. In our study, we have observed a conspicuous dose-dependent inhibition on the proliferation of synovial fibroblasts from RA patients after cells incubated with lunasin at gradually increasing concentrations. Moreover, we have observed that lunasin could decrease the secretion of IL- 6 in RA synovial fibroblasts in the presence of IL- $1 \beta$ induction.

In addition, the cartilage destruction of RA is mostly caused by the activity of MMPs, and MMPs are pivotal in the recruitment of leukocytes and macrophages into joints [38]. Our results showed that lunasin suppressed the secretion of MMP-3 but did not affect the secretion of MMP-1. It is generally accepted that proinflammatory cytokines such as IL-1, TNF- $\alpha$, and IL-6 are key mediators that greatly enhance the synthesis and secretion of MMPs [39]. In the present study, we have confirmed that lunasin can inhibit the secretion of IL- $1 \beta$-induced IL- 6 and IL- 8 in RA synovial fibroblasts. Therefore, the reduced secretion of IL- 6 and IL- 8 induced by lunasin may be partly responsible for the decrease of MMP-3 secretion.

Mitogen-activated protein kinase (MAPK) and NF- $\kappa \mathrm{B}$ pathways are involved in regulating the expression of IL- 6 , IL8, MMP-1, and MMP-3 in RA synovial fibroblasts $[6,8,40]$. According to one previous study, lunasin from soybean can inhibit the inflammation via the suppression of NF- $\kappa \mathrm{B}$ [34]. Consistent with other observations, our results clearly reveal that lunasin can suppress the activation of $\mathrm{NF}-\kappa \mathrm{B}$ induced by IL- $1 \beta$ in RA synovial fibroblasts. However, there were no direct data to confirm the association of inhibiting NF- $\kappa \mathrm{B}$ activation and reducing proinflammatory cytokines, which needs to be further explored in the future.

RA is the one of the most autoimmune diseases worldwide and thus the development of effective strategies for the prevention and therapy of this disease is highly desired. Based on our comprehensive and systematic exploration in cultured RA synovial fibroblasts, lunasin may have promising potential for the development of a novel and effective nutritional supplement or drug candidate for RA through inhibiting the proliferation of RA synovial fibroblasts via apoptosis 
activation, suppressing the expression of proinflammatory cytokines, and reducing the production of MMPs in RA synovial fibroblasts. The further studies on lunasin treatment for RA in animal models and in vivo need to be conducted too in the future, which will provide the benefit for relieving the suffering of RA patients.

\section{Abbreviations}

RGD: Arginine-glycine-aspartic acid

CDK2: Cyclin-dependent kinase 2

MTT: 3-(4,5-Dimethylthiazol-2-yl)-2,5diphenyltetrazolium bromide

DMEM: Dulbecco Modified Eagle Medium

ELISA: Enzyme linked immunosorbent assay

IL-6: Interleukin-6

IL-8: Interleukin-8

MMP: Matrix metalloproteinase

MAPK: Mitogen-activated protein kinase

NF- $\kappa$ B: Nuclear factor kappa-light-chain-enhancer of activated B cells

PI: $\quad$ Propidium iodide

RA: Rheumatoid arthritis.

\section{Conflict of Interests}

The authors have declared no conflict of interests.

\section{Acknowledgments}

The authors would like to thank Michael Kirberger from Department of Natural Science at Clayton State University in Georgia, USA, for providing the reviewing and suggestions of their paper. This project is financially supported by Chutian Scholar Program to Ning Chen from Education Department of Hubei Province, Innovation Program to Ning Chen from Wuhan Sports University, and Natural Science Foundation of Hubei Province (no. 2014CFB341) to Shaohui Jia from Science and Technology Department of Hubei Province.

\section{References}

[1] S. E. Gabriel and S. E. Gabriel, "The epidemiology of rheumatoid arthritis," Rheumatic Disease Clinics of North America, vol. 27, no. 2, pp. 269-281, 2001.

[2] S. E. Gabriel and K. Michaud, "Epidemiological studies in incidence, prevalence, mortality, and comorbidity of the rheumatic diseases," Arthritis Research and Therapy, vol. 11, no. 3, article 229, 2009.

[3] B. Bartok and G. S. Firestein, "Fibroblast-like synoviocytes: key effector cells in rheumatoid arthritis," Immunological Reviews, vol. 233, no. 1, pp. 233-255, 2010.

[4] G. S. Firestein, "Invasive fibroblast-like synoviocytes in rheumatoid arthritis: passive responders or transformed aggressors?" Arthritis \& Rheumatism, vol. 39, no. 11, pp. 1781-1790, 1996.

[5] M. Niedermeier, T. Pap, and A. Korb, "Therapeutic opportunities in fibroblasts in inflammatory arthritis," Best Practice and Research: Clinical Rheumatology, vol. 24, no. 4, pp. 527-540, 2010.
[6] P. P. Tak, D. M. Gerlag, K. R. Aupperle et al., "Inhibitor of nuclear factor kappaB kinase beta is a key regulator of synovial inflammation," Arthritis \& Rheumatology, vol. 44, pp. 1897-1907, 2001.

[7] C. Smith, E. Andreakos, J. B. Crawley, F. M. Brennan, M. Feldmann, and B. M. J. Foxwell, "NF- $\kappa$ B-inducing kinase is dispensable for activation of NF- $\kappa \mathrm{B}$ in inflammatory settings but essential for lymphotoxin $\beta$ receptor activation of NF- $\kappa \mathrm{B}$ in primary human fibroblasts," Journal of Immunology, vol. 167, no. 10, pp. 5895-5903, 2001.

[8] Z. Han, D. L. Boyle, L. Chang et al., "c-Jun N-terminal kinase is required for metalloproteinase expression and joint destruction in inflammatory arthritis," Journal of Clinical Investigation, vol. 108, pp. 73-81, 2001.

[9] C. Georganas, H. Liu, H. Perlman, A. Hoffmann, B. Thimmapaya, and R. M. Pope, "Regulation of IL-6 and IL-8 expression in rheumatoid arthritis synovial fibroblasts: the dominant role for NF-kappa B but not C/EBP beta or c-Jun," Journal of Immunology, vol. 165, no. 12, pp. 7199-7206, 2000.

[10] Z. Xu, G. Wu, X. Wei, X. Chen, Y. Wang, and L. Chen, "Celastrol induced DNA damage, cell cycle arrest, and apoptosis in human rheumatoid fibroblast-like synovial cells," The American Journal of Chinese Medicine, vol. 41, no. 3, pp. 615-628, 2013.

[11] D.-P. Chen, C.-K. Wong, P.-C. Leung et al., "Anti-inflammatory activities of Chinese herbal medicine sinomenine and Liang Miao San on tumor necrosis factor- $\alpha$-activated human fibroblast-like synoviocytes in rheumatoid arthritis," Journal of Ethnopharmacology, vol. 137, no. 1, pp. 457-468, 2011.

[12] D. Khanna, G. Sethi, K. S. Ahn et al., "Natural products as a gold mine for arthritis treatment," Current Opinion in Pharmacology, vol. 7, no. 3, pp. 344-351, 2007.

[13] T. Tokiwa, K. Harada, T. Matsumura, and T. Tukiyama, "Oriental medicinal herb, Periploca sepium, extract inhibits growth and IL-6 production of human synovial fibroblast-like cells," Biological and Pharmaceutical Bulletin, vol. 27, no. 10, pp. 16911693, 2004.

[14] N. Kusunoki, R. Yamazaki, H. Kitasato, M. Beppu, H. Aoki, and S. Kawai, "Triptolide, an active compound identified in a traditional Chinese herb, induces apoptosis of rheumatoid synovial fibroblasts," BMC Pharmacology, vol. 4, article 2, 2004.

[15] B. Hernández-Ledesma, C.-C. Hsieh, and B. O. de Lumen, "Lunasin, a novel seed peptide for cancer prevention," Peptides, vol. 30, no. 2, pp. 426-430, 2009.

[16] A. F. Galvez and B. O. de Lumen, "A soybean cDNA encoding a chromatin-binding peptide inhibits mitosis of mammalian cells," Nature Biotechnology, vol. 17, no. 5, pp. 495-500, 1999.

[17] S. E. D'Souza, M. H. Ginsberg, and E. F. Plow, "Arginylglycyl-aspartic acid (RGD): a cell adhesion motif," Trends in Biochemical Sciences, vol. 16, no. 1, pp. 246-250, 1991.

[18] A. F. Galvez, N. Chen, J. Macasieb, and B. O. de Lumen, "Chemopreventive property of a soybean peptide (Lunasin) that binds to deacetylated histones and inhibits acetylation," Cancer Research, vol. 61, no. 20, pp. 7473-7478, 2001.

[19] H. J. Jeong, J. B. Jeong, D. S. Kim et al., "The cancer preventive peptide lunasin from wheat inhibits core histone acetylation," Cancer Letters, vol. 255, no. 1, pp. 42-48, 2007.

[20] J. Liu, S. H. Jia, M. Kirberger, and N. Chen, "Lunasin as a promising health-beneficial peptide," European Review for Medical and Pharmacological Sciences, vol. 18, pp. 2070-2075, 2014.

[21] C. C. Hsieh, B. Hernández-Ledesma, and B. O. de Lumen, "Lunasin, a novel seed peptide, sensitizes human breast cancer 
MDA-MB-231 cells to aspirin-arrested cell cycle and induced apoptosis," Chemico-Biological Interactions, vol. 186, no. 2, pp. 127-134, 2010.

[22] V. P. Dia, M. A. Berhow, and E. G. de Mejia, "Bowman-birk inhibitor and genistein among soy compounds that synergistically inhibit nitric oxide and prostaglandin E2 pathways in lipopolysaccharide-induced macrophages," Journal of Agricultural and Food Chemistry, vol. 56, no. 24, pp. 11707-11717, 2008.

[23] B. Hernández-Ledesma, C.-C. Hsieh, and B. O. de Lumen, "Antioxidant and anti-inflammatory properties of cancer preventive peptide lunasin in RAW 264.7 macrophages," Biochemical and Biophysical Research Communications, vol. 390, no. 3, pp. 803-808, 2009.

[24] S. Liu, W.-Y. Tan, Q.-R. Chen et al., "Daintain/AIF-1 promotes breast cancer proliferation via activation of the NFkappaB/cyclin D1 pathway and facilitates tumor growth," Cancer Science, vol. 99, no. 5, pp. 952-957, 2008.

[25] R. W. Kinne, E. Palombo-Kinne, and F. Emmrich, "Activation of synovial fibroblasts in rheumatoid arthritis," Annals of the Rheumatic Diseases, vol. 54, no. 6, pp. 501-504, 1995.

[26] G. S. Firestein, M. Yeo, and N. J. Zvaifler, "Apoptosis in rheumatoid arthritis synovium," Journal of Clinical Investigation, vol. 96, no. 3, pp. 1631-1638, 1995.

[27] R. M. Pope, "Apoptosis as a therapeutic tool in rheumatoid arthritis," Nature Reviews Immunology, vol. 2, no. 7, pp. 527-535, 2002.

[28] K. Matsuki, T. Sasho, K. Nakagawa et al., "RGD peptideinduced cell death of chondrocytes and synovial cells," Journal of Orthopaedic Science, vol. 13, no. 6, pp. 524-532, 2008.

[29] C. D. Anuradha, S. Kanno, and S. Hirano, "RGD peptideinduced apoptosis in human leukemia HL-60 cells requires caspase-3 activation," Cell Biology and Toxicology, vol. 16, no. 5, pp. 275-283, 2000.

[30] V. P. Dia and E. G. D. Mejia, "Lunasin promotes apoptosis in human colon cancer cells by mitochondrial pathway activation and induction of nuclear clusterin expression," Cancer Letters, vol. 295, no. 1, pp. 44-53, 2010.

[31] M. Ohtsubo, A. M. Theodoras, J. Schumacher, J. M. Roberts, and M. Pagano, "Human cyclin E, a nuclear protein essential for the G1-to-S phase transition," Molecular and Cellular Biology, vol. 15, no. 5, pp. 2612-2624, 1995.

[32] D. A. Foster, P. Yellen, L. Xu, and M. Saqcena, "Regulation of G1 cell cycle progression: distinguishing the restriction point from a nutrient-sensing cell growth checkpoint(s)," Genes and Cancer, vol. 1, no. 11, pp. 1124-1131, 2011.

[33] S. A. Ezhevsky, A. Ho, M. Becker-Hapak, P. K. Davis, and S. F. Dowdy, "Differential regulation of retinoblastoma tumor suppressor protein by G1 cyclin-dependent kinase complexes in vivo," Molecular and Cellular Biology, vol. 21, no. 14, pp. 47734784, 2001.

[34] E. G. de Mejia and V. P. Dia, "Lunasin and lunasin-like peptides inhibit inflammation through suppression of NF- $\kappa$ B pathway in the macrophage," Peptides, vol. 30, no. 12, pp. 2388-2398, 2009.

[35] C. Moon, J. R. Han, H.-J. Park, J. S. Hah, and J. L. Kang, "Synthetic RGDS peptide attenuates lipopolysaccharide-induced pulmonary inflammation by inhibiting integrin signaled MAP kinase pathways," Respiratory Research, vol. 10, article 18, 2009.

[36] N. Nishimoto, A. Ito, M. Ono et al., "IL-6 inhibits the proliferation of fibroblastic synovial cells from rheumatoid arthritis patients in the presence of soluble IL-6 receptor," International Immunology, vol. 12, no. 2, pp. 187-193, 2000.
[37] M. Mihara, Y. Moriya, T. Kishimoto, and Y. Ohsugi, "Interleukin-6 (IL-6) induces the proliferation of synovial fibroblastic cells in the presence of soluble IL-6 receptor," British Journal of Rheumatology, vol. 34, no. 4, pp. 321-325, 1995.

[38] M. Feldmann, F. M. Brennan, and R. N. Maini, "Role of cytokines in rheumatoid arthritis," Annual Review of Immunology, vol. 14, pp. 397-440, 1996.

[39] J. F. Woessner Jr., "Matrix metalloproteinases and their inhibitors in connective tissue remodeling," The FASEB Journal, vol. 5, no. 8, pp. 2145-2154, 1991.

[40] K. R. Aupperle, B. L. Bennett, Z. Han, D. L. Boyle, A. M. Manning, and G. S. Firestein, "NF- $\kappa$ B regulation by $\mathrm{I} \kappa \mathrm{B}$ kinase2 in rheumatoid arthritis synoviocytes," Journal of Immunology, vol. 166, no. 4, pp. 2705-2711, 2001. 

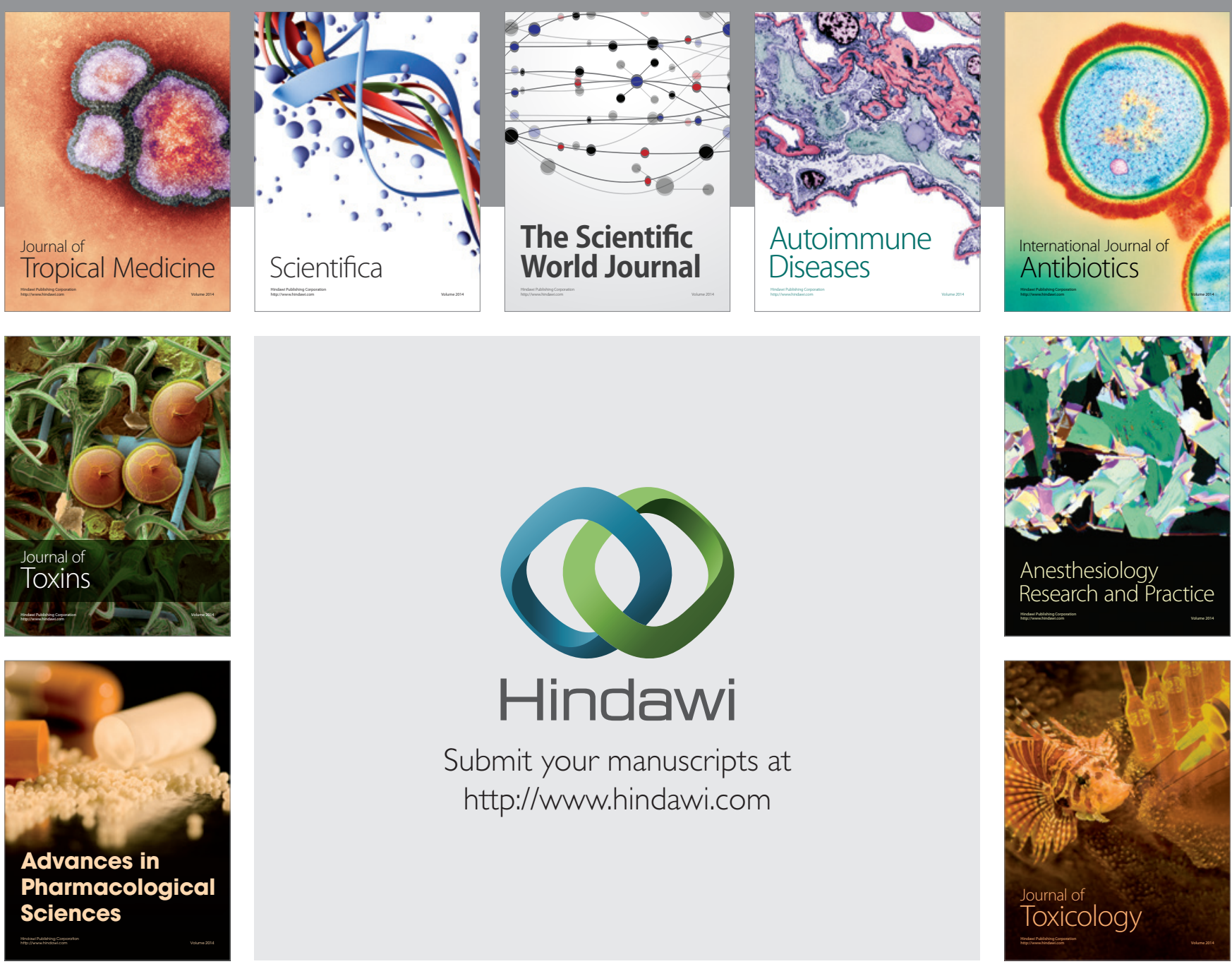

\section{Hindawi}

Submit your manuscripts at

http://www.hindawi.com
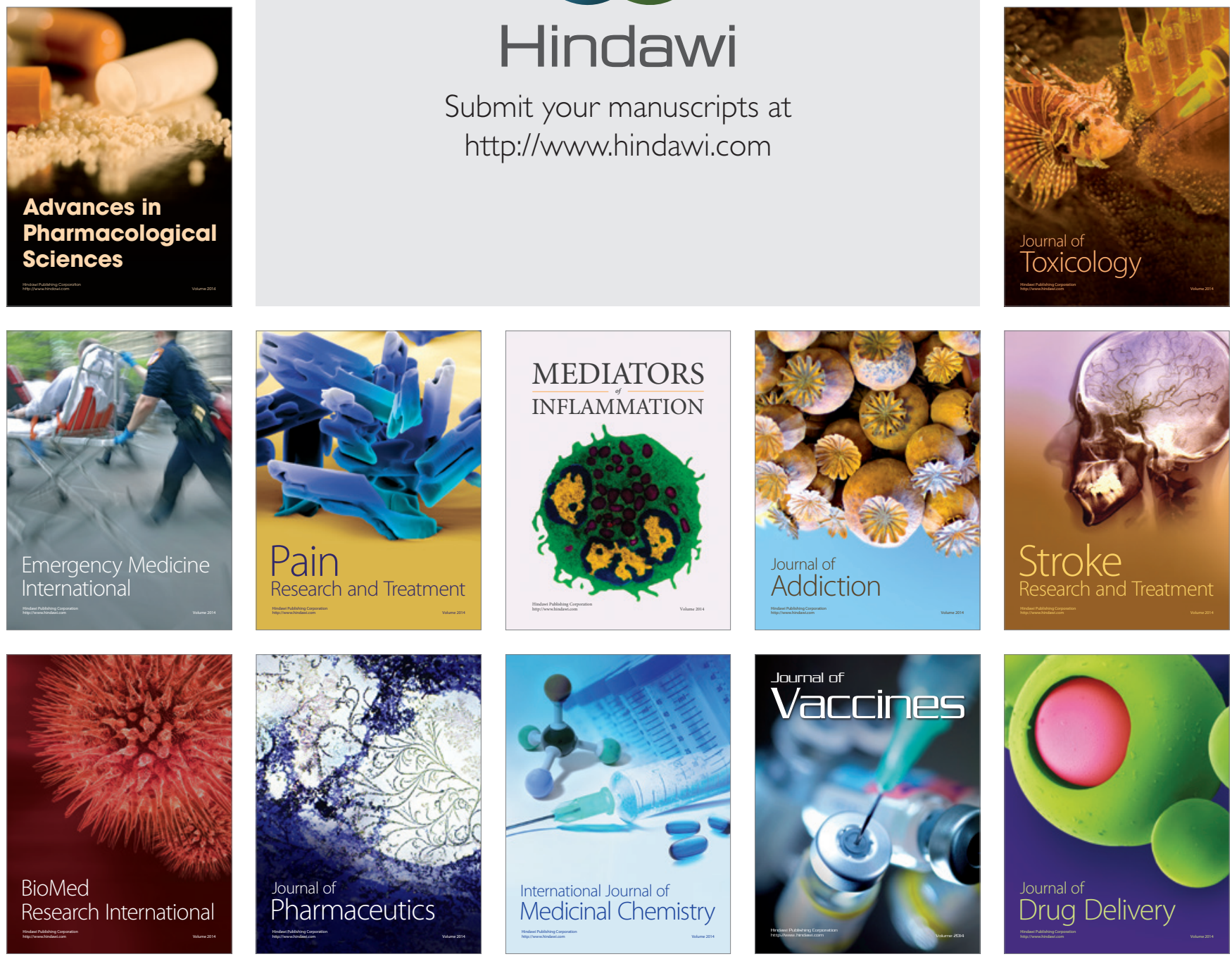